\title{
Emerging outcome measures for nutrition trials in the critically ill
}

\author{
Danielle E. Bear ${ }^{\mathrm{a}, \mathrm{b}, \mathrm{c}, \mathrm{d}, *}$, David Griffith $^{\mathrm{e}, *}$, and Zudin A. Puthucheary ${ }^{\mathrm{d}, \mathrm{f}, \mathrm{g}, *}$
}

\begin{abstract}
Purpose of review
Mortality has long been the gold-standard outcome measure for intensive care clinical trials. However, as the critical care community begins to understand and accept that survivorship is associated with functional disability and a health and socioeconomic burden, the clinical and research focus has begun to shift towards long-term physical function
\end{abstract}

\begin{abstract}
Recent findings
To use mortality as a primary outcome measure, one would either have to choose an improbable effect (e.g. a difference of $5-10 \%$ in mortality as a result of a single intervention) or recruit a larger number of patients, the latter being unfeasible for most critical care trials.

Outcome measures will need to match interventions. As an example, amino acids, or intermittent feeding, can stimulate muscle protein synthesis, and so prevention of muscle wasting may seem an appropriate outcome measure when assessing the effectiveness of these interventions. Testing the effectiveness of these interventions requires the development of novel outcome measures that are targeted and acceptable to patients. We describe advancements in dual-energy X-ray absorptiometry scanning, bio-impedence analysis, MRI and muscle ultrasound in this patient group that are beginning to address this development need.
\end{abstract}

\begin{abstract}
Summary
New approaches to outcome assessment are beginning to appear in post-ICU research, which promise to improve our understanding of nutrition and exercise interventions on skeletal muscle structure, composition and function, without causing undue suffering to the patient.
\end{abstract}

\section{Keywords}

anabolism, functional outcomes, imaging, muscle

\section{INTRODUCTION}

Mortality has long been the gold-standard outcome measure for intensive care clinical trials - this being the ultimate rationale behind the use of multi-organ support in the sickest patients [1]. However, hospital and intensive care mortality are perhaps no longer considered the best time points, with 90-day, 6month and 1-year mortality being examined [2]. Additionally, acquired morbidity following critical illness is of increasing interest [3].

The changing face of intensive care medicine has ramifications for clinical trial design and methodology, including trials investigating nutrition interventions. In the setting of modern critical care, an unrefined patient population will have an ICU mortality of $20 \%$, rising to closer to $30 \%$ at hospital discharge [4]. To use mortality as a primary outcome measure, one would either have to choose an improbable effect (e.g. a difference of $5-10 \%$ in mortality as a result of a single intervention) or recruit a larger number of patients, the latter being unfeasible for most critical care trials [5]. However, equipoise exists as to whether or not a single intervention can affect mortality in a population in which multiple physiological stressors (e.g. inflammation, hypoxia, multi-organ failure) will

${ }^{a}$ Department of Nutrition and Dietetics, ${ }^{b}$ Department of Critical Care, 'Lane Fox Clinical Respiratory Research Unit, Guy's and St Thomas' NHS Foundation Trust, ${ }^{\mathrm{d}}$ Centre for Human and Applied Physiological Sciences, King's College London, ${ }^{\mathrm{e} A n a e s t h e s i a, ~ C r i t i c a l ~ C a r e ~ a n d ~ P a i n ~}$ Medicine, The University of Edinburgh, Edinburgh, ${ }^{f}$ Centre for Human Health and Performance, Department of Medicine, University College London and ${ }^{9}$ Adult Intensive Care Unit, Royal Free Hospital NHS Foundation Trust London, London, UK

Correspondence to Zudin A. Puthucheary, Institute of Sport and Exercise Health, 1st Floor, 170 Tottenham Court Road, London W1T 7HA, UK. E-mail: Zudin.puthucheary.09@ucl.ac.uk

${ }^{*}$ All authors contributed equally.

Curr Opin Clin Nutr Metab Care 2018, 21:417-422

DOI:10.1097/MCO.0000000000000507 


\section{KEY POINTS}

- The focus of critical care research is shifting from survival to long-term physical function.

- Innovative nutrition interventions are likely to have a pivotal role in maintaining neuromuscular function during critical illness and restoring it afterwards.

- Testing the effectiveness of these interventions requires the development of novel outcome measures that are targeted and acceptable to patients.

- We describe advancements in DXA scanning, bioimpedence analysis, MRI and muscle ultrasound in this patient group that are beginning to address this development need.

contribute. This scenario is possibly fuelled by large multicentre observational studies demonstrating positive associations between single interventions, such as nutritional delivery [6]. However, the recently completed TARGET study (NCT02306746, The Augmented versus Routine approach to Giving Energy Trial) did indeed recruit a large number of patients (4000) and may offer a potential answer on causality.

As a result of the growing interest in survivorship, critical care trials are increasingly either embedding health-related quality of life and/or functional outcome measures as secondary outcomes, or using these measures as primary outcomes $[7,8]$. Considering these outcomes, nutritional interventions are plausible as appropriate, effective interventions. Amino acid availability affects protein homeostasis and thus muscle mass. Substrates for energy production are necessary for protein homeostasis and for muscle (and indeed all cells) to function [9]. Thus, nutritional interventions may indeed affect functional and health-related outcomes by affecting muscle mass and function, but limited data exist to support this.

\section{SIGNAL-TO-NOISE RATIOS}

Several recent trials have incorporated measures of muscle mass or physical or functional outcomes into their study design, albeit mainly as secondary outcomes or in smaller sub-studies, with varying results $\left[10,11^{*}, 12\right]$. As an example, early goal directed nutrition in ICU patients (EAT-ICU) [11"] aimed to investigate the effect of early goal-directed nutrition (EGDN) over the first week of ICU stay on the Physical Component Score (PCS) of the short form (36) health survey at 6 months. Protein intakes were targeted based on urinary urea nitrogen measurements, and adjusted according to plasma urea. No differences in the primary or secondary outcomes were found. Such studies lead clinicians to believe that nutrition support does not enhance physical or functional outcomes. This may or may not be true, but it is not clear that such studies (no matter how well executed) contribute to this understanding. Long-term outcomes are affected by a multitude of factors preceding critical illness (such as frailty, comorbidities and socio-economic status), and post critical illness (such as persistent inflammation, new organ dysfunction and post-traumatic stress disorders) [13"]. To statistically dissect out the signal of a 5 or 10-day intervention on 6-month to 1year outcomes through this noise would seem challenging if not nearly impossible, regardless of the intervention [14]. Several post-hoc analyses of physical rehabilitation trials have demonstrated the importance of baseline correction for outcome [15"] and stratification by post-critical illness biology [16"] as methods to refine data for outcome analyses.

Two newer concepts are likely to be explored in future critical care trials. First, 'critical illness' is currently being defined by time epochs - 'acute critical illness', 'chronic critical illness [17]' and 'post-intensive care syndrome [18]'. However, these epochs lack markers of division where these markers are far more likely to be biological as opposed to merely time-based (e.g. markers of inflammation versus days since admission). This is particularly pertinent when considering trials of nutrition and exercise where the biological interaction between these interventions may be uncoupled early in critical illness [19], and are likely to only be effective once this is re-coupled [20]. Work has begun within the epoch between ICU discharge and hospital discharge, an area with little data and where interventions are far more likely to be effective as the confounders of inflammation, hypoxia and multiorgan failure have subsided [21,22]. We now know that, after discharge, our patients continue through their hospital admission not meeting energy or protein targets [23], which is likely to have a significant impact on outcome and needs to be accounted for.

Second, outcome measures will need to match interventions. As an example, amino acids, or intermittent feeding, can stimulate muscle protein synthesis, and so prevention of muscle wasting may seem an appropriate outcome measure when assessing the effectiveness of these interventions. For muscle to gain mass and quality (and therefore increase function) a combination of amino acids and exercise is required. A functional outcome may therefore be appropriate [24]. The difficulty 
here is that this is an emerging aspect of critical care nutritional research, and therefore knowledge translation from other patient groups is necessary.

\section{UNDERSTANDING METABOLIC DYSFUNCTION IS ESSENTIAL FOR SUCCESSFUL OUTCOME DEVELOPMENT}

Much work has been done in understanding the unique factors underpinning measurement instruments of physical function in trials of rehabilitation in critical illness [25]. An important element that has come out of this work is the importance of considering an instrument that matches the intended outcome and the intervention. Much less work has been done in the field of nutrition, and although the importance of including physical or functional outcomes in trials of nutrition support in critical illness is accepted, much less is understood about the underlying physiology of these interventions how they will lead to the desired outcome [26]. It is likely that different interventions, and therefore, perhaps, outcome measures, will be most useful at different time epochs during admission to match the dynamic changes in physiology (Fig. 1).

Starting from admission, baseline characteristics play an important role in the likelihood of a successful intervention. Patients may enter the ICU malnourished [27] or with low muscle mass [28], with several comorbidities or of an advanced age, all of which not only influence functional performance and ability $[7,29]$, but response to nutrition [30]. The amount of muscle mass may influence requirements for nutrients such as protein; however, current practice is to set targets based on bodyweight (actual or ideal) as opposed to body composition (e.g. amount of lean mass). It would not seem unreasonable to suggest that nutrition targets should be based on the amount muscle mass if functional outcomes are to be measured.

Throughout the first week of ICU stay it has been shown that muscle protein synthesis is initially depressed to levels associated with fasted controls, with variable recovery over the following 7 days, but muscle protein catabolism remains raised, leading to a net catabolic balance and the subsequent loss of skeletal muscle [31]. New data suggest that that muscle protein balance becomes less negative over time, with a plateau at about day 35 [32"'], importantly, driven mainly by an increase in muscle protein synthesis. These data support the suggestion that nutrition interventions should be tailored to the time point of admission according to the physiological changes that are occurring. For example, specific amino acid metabolites, such as $\beta$-hydroxy$\beta$-methylbutyrate (HMB), which target catabolic pathways [33], may be efficacious in the early phases of ICU admission. This may be followed by administration of amino acids such as leucine, that target the pathways of muscle protein synthesis [34], in the latter phases of critical illness to tip the balance into a more anabolic state.

Underpinning all of the above is the contribution of inflammation and generation of ATP. Both inflammation and lack of ATP inhibit muscle protein synthesis, which may be a limiting factor for

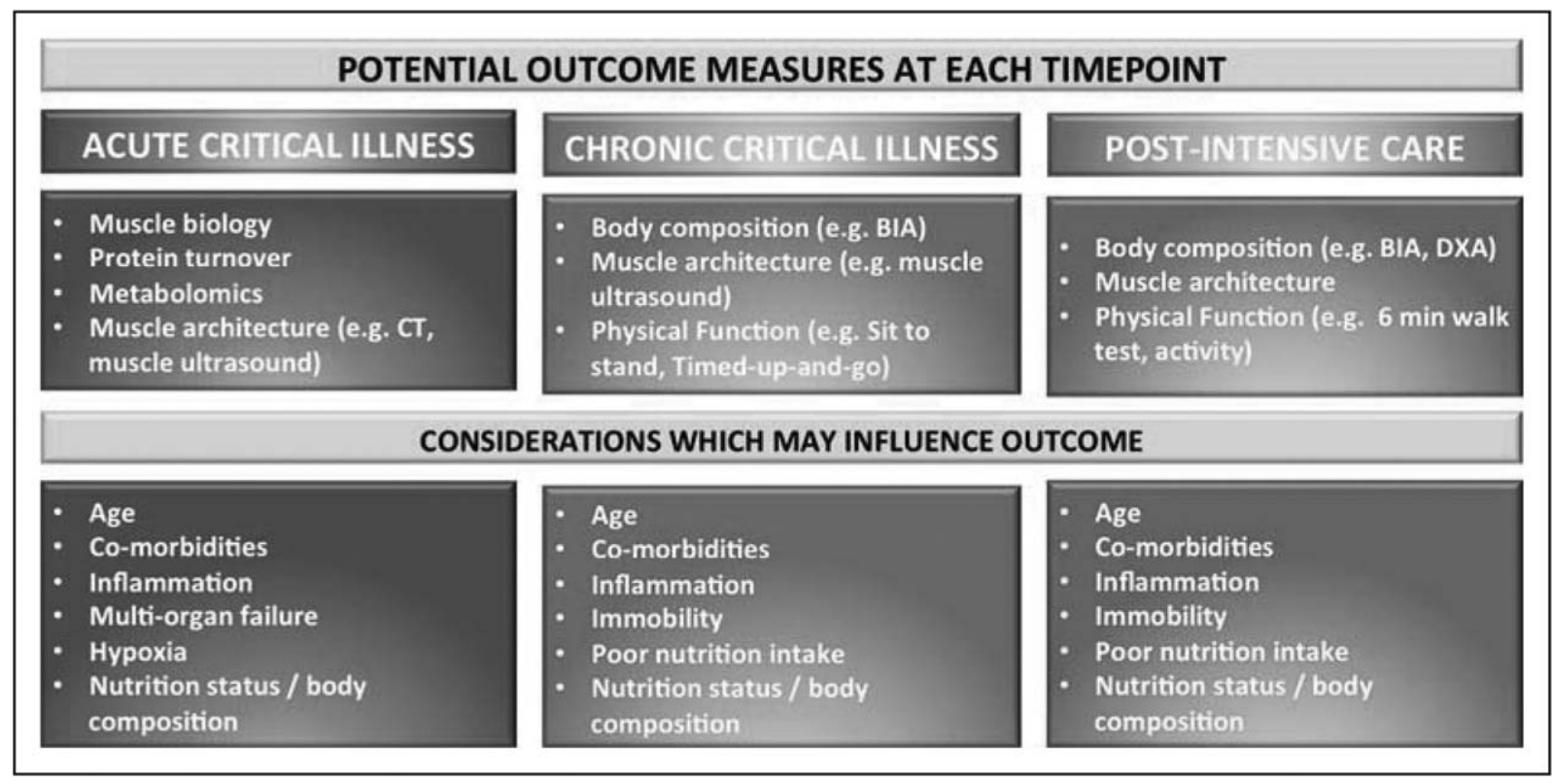

FIGURE 1. Potential outcome measures and considerations which may influence outcome at each time point during critical illness. BIA, bioelectrical impedance analysis; CT, computed tomography; DXA, dual-energy X-ray absorptiometry. 
any intervention aiming to reduce skeletal muscle wasting and enhance physical and functional performance [35"']. Considering nutrition interventions that modulate these, such as HMB or inducing ketosis to provide an alternative fuel source for ATP generation, may be warranted [9].

Lastly, the combined effect of nutrition and exercise requires consideration [36], though implementation of this strategy is not without difficulty, as there are several factors which may influence functional ability including muscle endurance, fatigability, cognition, mental health, sleep disturbance along with the patients' premorbid health, age and length of illness $[7,25,29]$. Nutrition strategies to combine with different exercise regimens require careful consideration of the mechanisms underlying their potential effect. Data from populations outside of critical illness may provide important insights. For example, coupling exercise with bolus/intermittent feeding to enhance the anabolic window seen with the 'muscle full effect' in healthy individuals may be one intervention worth considering.

\section{MUSCLE MASS-BASED OUTCOME MEASURES IN THE CRITICAL ILLNESS SURVIVOR}

Although our understanding of the physical problems faced by ICU survivors continues to increase, the gulf in the literature between what happens to skeletal muscle during and after acute critical illness is significant. Although critically unwell, patients remain within convenient proximity of the researchers, and unpleasant procedures such as muscle biopsy can be conducted under analgo-sedation or anaesthesia, lessening the inconvenience and discomfort experienced by the individual. Once discharged to the community, follow-up is far less complete [37]. Our understanding of what happens to skeletal muscle after hospital discharge is often derived indirectly from outcome measures that can be assessed reliably by telephone interview or survey as seen in the recent EAT-ICU trial [11"].

It is in smaller-scale observational studies exploring the mechanisms of weakness and recovery that the more proximal and organ-specific measures can be found. Even blood sampling suffers significantly greater attrition when compared to other outcome measures [16"]. It seems unlikely that participants who feel able to return to a healthcare facility for detailed testing are truly representative of a population that may also contain individuals who, for physical or psychological reasons, cannot attend. Unfortunately, our most detailed studies of muscular regeneration after ICU may have so far mainly been conducted in the fittest of ICU survivors. New approaches to outcome assessment are beginning to appear in post-ICU research, which promise to improve our understanding of nutrition and exercise interventions on skeletal muscle structure, composition and function, without causing undue suffering to the patient.

In the sub-study of patients with acute respiratory distress syndrome mentioned above, Chan [38] employed dual-energy X-ray absorptiometry (DXA) to study body composition after ICU discharge, participants tended to gain fat mass while maintaining lean (skeletal muscle) mass. Although strength and self-reported function were not affected by body composition, walking speed and distance were improved in patients who had a higher muscle-tofat ratio, perhaps revealing an important link between body composition and physical function [38].

As a nutrition outcome measure, DXA scanning is therefore appealing. It is acceptable to patients, is conducted with a very low dose of ionizing radiation and provides information about both fat and muscle mass. This latter benefit is particularly relevant to studies of nutrition interventions where improvement of lean mass, in specific preference to an increase in fat mass, is often the goal.

A continuous improvement in the portability and resolution of musculoskeletal ultrasound (US) makes it attractive as a non-invasive measure of muscle volume and quality during follow-up from ICU, and may be the ideal modality for assessing the impact of nutrition interventions during and after ICU admission. Measurements of muscle mass, quality and architectural structure have been embraced [39]. Despite a significant variation in the conduct and reporting of these studies, and a requirement for standardization of approaches [40,41], the emergence of novel US measures, such as measures of glycogen storage, may have relevance in the postICU period, despite having limitations in the acutely unwell patient. Surprisingly, the application of musculoskeletal US in the post-ICU period has been limited. In a 37-patient study of ICU patients with traumatic brain injury (TBI), quadriceps muscle layer thickness (QMLT) was assessed in the postICU ward after ICU discharge and at 3 months post-ICU discharge. Eight of the included patients underwent DXA scanning, and in these patients, total body lean mass was strongly associated with QMLT, suggesting perhaps a role for post-ICU US for assessment of muscle quality. Despite this, like many other post-ICU studies, there was a major attrition, with only 13 (35\%) of the original sample available for US at 3 months [27].

Body composition can be also estimated noninvasively using bioelectrical impedance (BIA). In 
healthy individuals, BIA can reliably estimate fatfree mass (FFM) by applying regression equations to measured BIA parameters. These estimates are not accurate in patients with deranged physiology, where fluid compartments are dynamic such as in critical illness [42]. Raw values such as phase angle may be useful for predicting outcome in certain disease states, but for tracking nutrition during critical illness are unlikely to be useful, as changes may reflect alterations in fluid status rather than nutrition or muscle mass. In the post-ICU period, there may be value in studying phase angle over time, and if validated against a radiological measure such as intramuscular fat fraction, may have significant value as a marker of nutrition recovery.

Measurements of muscle cross-sectional area and density, reflecting total body muscle mass and quality, have been measured in diagnostic computed tomography (CT) scans acquired during critical illness [39]. Low muscle mass and quality are associated with negative outcomes, such as mortality and length of ICU treatment $[39,43]$, but even in the hospital setting, the drawbacks of cost, time, transport risk and radiation exposure preclude prospective evaluation. Although the transport risks in the post-ICU patient are minimal, the other drawbacks remain significant, and CT has not yet been used to track muscle recovery after hospital discharge. However, given the use of CT to diagnose and monitor a significant number of other factors which influence patient morbidity, it is perhaps not unreasonable to begin to consider the use of inhospital CT scans to monitor body composition and allow treatments to be tailored to the individual.

Similarly, MRI provides an alternative to muscle biopsy in the study of myopathy. In contrast to radiological techniques, MRI can identify muscle abnormalities within specific muscle compartments identifying areas of oedema (normally associated with acute processes) and lipid infiltration (normally associated with chronic process). It will also undoubtedly be useful for delineating the precise location of fat (intra versus extra-muscular) in body composition studies. Although impractical in the assessment of acutely unwell patients, its feasibility in the post-ICU period is currently being explored (Griffith, personal communication; trial registry ISCTRN1782997). Whilst likely to be useful in exploring mechanisms of muscular recovery, its importance as an outcome measure in ICU nutrition trials is currently limited by its cost and the unclear relationship to physical outcomes. Further research is required to understand whether a reduction in oedema or an improvement in muscle quality translates into an improvement in health.

\section{CONCLUSION}

Nutritional research in critical illness continues to evolve. To maintain clinical relevance, outcome measures related to function and muscle mass are likely to be increasingly important as opposed to mortality. Newer outcomes are needed that map to the increasingly diverse and complex interventions seen in trials.

\section{Acknowledgements}

None.

\section{Financial support and sponsorship}

None.

\section{Conflicts of interest}

Z.P. has received honoraria for consultancy from GlaxoSmithKleine, Lyric Pharmaceuticals and FreseniusKabi and speaker fees from Orion. D.E.B. has received speaker fees and conference attendance support from Nutricia, Baxter, BBraun, Nestle Nutrition and Fresenius Kabi; Consultancy fees through her institution from Abbott Nutrition and grant support through her institution from Corpak MedSystems UK.

\section{REFERENCES AND RECOMMENDED}

\section{READINC}

Papers of particular interest, published within the annual period of review, have been highlighted as:

- of special interest

m. of outstanding interest

1. Turnbull AE, Rabiee A, Davis WE, et al. Outcome measurement in ICU survivorship research from 1970 to 2013: a scoping review of 425 publications. Crit Care Med 2016; 44:1267-1277.

2. Puthucheary Z, Prescott $H$. Skeletal muscle weakness is associated with both early and late mortality after acute respiratory distress syndrome. Crit Care Med 2017; 45:563-565.

3. Ruhl AP, Huang M, Colantuoni $\mathrm{E}$, et al. Healthcare resource use and costs in long-term survivors of acute respiratory distress syndrome: a 5-year longitudinal cohort study. Crit Care Med 2017; 45:196-204.

4. ICNARC. https://www.icnarc.org/Our-Audit/Audits/Cmp/Reports/SummaryStatistics.

5. Harhay MO, Wagner J, Ratcliffe SJ, et al. Outcomes and statistical power in adult critical care randomized trials. Am J Respir Crit Care Med 2014; 189:1469-1478.

6. Compher C, Chittams J, Sammarco T, et al. Greater protein and energy intake may be associated with improved mortality in higher risk critically ill patients: a multicenter, multinational observational study. Crit Care Med 2017; 45:156-163.

7. Latronico N, Herridge M, Hopkins RO, et al. The ICM research agenda on intensive care unit-acquired weakness. Intensive Care Med 2017; 43:1270-1281

8. Arabi YM, Casaer MP, Chapman M, et al. The intensive care medicine research agenda in nutrition and metabolism. Intensive Care Med 2017; 43:1239-1256.

9. Bear DE, Parry SM, Puthucheary ZA. Can the critically ill patient generate sufficient energy to facilitate exercise in the ICU? Curr Opin Clin Nutr Metab Care 2018; 21:110-115.

10. Ferrie $S$, Allman-Farinelli $M$, Daley $M$, Smith $K$. Protein requirements in the critically ill: a randomized controlled trial using parenteral nutrition. JPEN J Parenter Enteral Nutr 2016; 40:795-805.

11. Allingstrup MJ, Kondrup J, Wiis J, et al. Early goal-directed nutrition versus

- standard of care in adult intensive care patients: the single-centre, randomised, outcome assessor-blinded EAT-ICU trial. Intensive Care Med 2017; 43:1637-1647.

A nutritional intervention trial in which a short period of intervention was expected to alter long-term outcomes. 
12. Fetterplace $K$, Deane $A M$, Tierney $A$, et al. Targeted full energy and protein delivery in critically ill patients: a pilot randomized controlled trial (FEED trial). JPEN J Parenter Enteral Nutr 2018; doi: 10.1002/jpen.1166 [Epub ahead of print]

13. Griffith DM, Salisbury LG, Lee RJ, et al. Determinants of health-related quality

- of life after ICU: importance of patient demographics, previous comorbidity, and severity of illness. Crit Care Med 2018; 46:594-601.

This study summarizes many of the often ignored factors that affect long-term outcomes.

14. Wright SE, Thomas $\mathrm{K}$, Watson $\mathrm{G}$, et al. Intensive versus standard physical rehabilitation therapy in the critically ill (EPICC): a multicentre, parallel-group, randomised controlled trial. Thorax 2018; 73:213-221.

15. Puthucheary ZA, Denehy L. Exercise interventions in critical illness survivors: understanding inclusion and stratification criteria. Am J Respir Crit Care Med $2015 ; 191: 1464-1467$.

This is the first description of how pre-existing diseases can affect randomized controlled trials.

16. Griffith DM, Lewis $S$, Rossi $A G$, et al. Systemic inflammation after critical

- illness: relationship with physical recovery and exploration of potential mechanisms. Thorax 2016; 71:820-829.

This is the first study to suggest a causal link between inflammation and persistent functional disability.

17. Efron PA, Mohr AM, Bihorac A, et al. Persistent inflammation, immunosuppression, and catabolism and the development of chronic critical illness after surgery. Surgery 2018; 164:178-184.

18. Marra A, Pandharipande PP, Girard TD, et al. Cooccurrence of post-intensive care syndrome problems among 406 survivors of critical illness. Crit Care Med 2018; doi: 10.1097/CCM.000000000000321 [Epub ahead of print]

19. Connolly B, Maddocks M, MacBean V, et al. Nonvolitional assessment of tibialis anterior force and architecture during critical illness. Muscle Nerve 2018; 57:964-972.

20. Wischmeyer PE, Puthucheary Z, San Millán I. Muscle mass and physical recovery in ICU: innovations for targeting of nutrition and exercise. Curr Opin Crit Care 2017; 23:269-278.

21. Merriweather JL, Griffith DM, Walsh TS. Appetite during the recovery phase of critical illness: a cohort study. Eur J Clin Nutr 2018; 72:986-992.

22. Merriweather JL, Salisbury LG, Walsh TS, Smith $P$. Nutritional care after critical illness: a qualitative study of patients' experiences. J Hum Nutr Diet 2016; 29:127-136.

23. Chapple LS, Deane AM, Heyland DK, et al. Energy and protein deficits throughout hospitalization in patients admitted with a traumatic brain injury. Clin Nutr 2016; 35:1315-1322.

24. Parry $S M$, Nydahl $P$, Needham $D M$. Implementing early physical rehabilitation and mobilisation in the ICU: institutional, clinician, and patient considerations. Intensive Care Med 2018; 44:470-473.

25. Parry SM, Huang M, Needham DM. Evaluating physical functioning in critical care: considerations for clinical practice and research. Crit Care 2017;21:249.

26. Bear DE, Wandrag $L$, Merriweather JL, et al. The role of nutritional support in the physical and functional recovery of critically ill patients: a narrative review. Crit Care 2017; 21:226.

27. Chapple LS, Deane AM, Williams LT, et al. Longitudinal changes in anthropometrics and impact on self-reported physical function after traumatic brain injury. Crit Care Resusc 2017; 19:29-36.
28. Weijs PJ, Looijaard WG, Dekker IM, et al. Low skeletal muscle area is a risk factor for mortality in mechanically ventilated critically ill patients. Crit Care 2014; 18:R12.

29. Herridge MS, Chu LM, Matte A, et al. The RECOVER program: disability risk groups and 1-year outcome after 7 or more days of mechanical ventilation. Am J Respir Crit Care Med 2016; 194:831-844.

30. Moore DR, Churchward-Venne TA, Witard O, et al. Protein ingestion to stimulate myofibrillar protein synthesis requires greater relative protein intakes in healthy older versus younger men. J Gerontol A Biol Sci Med Sci 2015; 70:57-62.

31. Puthucheary ZA, Rawal J, McPhail M, et al. Acute skeletal muscle wasting in critical illness. JAMA 2013; 310:1591-1600.

32. Gamrin-Gripenberg L, Sundström-Rehal M, Olsson D, et al. An attenuated

-1 rate of leg muscle protein depletion and leg free amino acid efflux over time is seen in ICU long-stayers. Crit Care 2018; 22:13.

This is one of only two studies demonstrating muscle protein synthesis as the main driver of altered muscle protein homeostasis in critically ill patients.

33. Wilkinson DJ, Hossain T, Limb MC, et al. Impact of the calcium form of beta-hydroxy-beta-methylbutyrate upon human skeletal muscle protein metabolism. Clin Nutr 2017; doi: 10.1016/j.clnu.2017.09.024 [Epub ahead of print]

34. Mitchell WK, Wilkinson DJ, Phillips BE, et al. Human skeletal muscle protein metabolism responses to amino acid nutrition. Adv Nutr 2016; $7: 828 \mathrm{~S}-838 \mathrm{~S}$

35. Puthucheary ZA, Astin R, Mcphail MJW, et al. The metabolic phenotype of

- skeletal muscle is acute critical illness. Thorax 2018; doi: 10.1136/thoraxjnl2017-211073 [Epub ahead of print]

This is the first physiological study to link inflammation, low ATP content and altered lipid metabolism in critically ill patients.

36. Heyland DK, Stapleton RD, Mourtzakis M, et al. Combining nutrition and exercise to optimize survival and recovery from critical illness: conceptual and methodological issues. Clin Nutr 2016; 35:1196-1206.

37. Dos Santos C, Hussain SN, Mathur S, et al. Mechanisms of chronic muscle wasting and dysfunction after an intensive care unit stay. A pilot study. Am J Respir Crit Care Med 2016; 194:821-830.

38. Chan KS, Aronson Friedman L, Dinglas VD, et al. Are physical measures related to patient-centred outcomes in ARDS survivors? Thorax 2017; 72:884-892.

39. Looijaard W, Molinger J, Weijs PJM. Measuring and monitoring lean body mass in critical illness. Curr Opin Crit Care 2018; 24:241-247.

40. Mourtzakis M, Parry S, Connolly B, Puthucheary Z. Skeletal muscle ultrasound in critical care: a tool in need of translation. Ann Am Thorac Soc 2017; $14: 1495-1503$.

41. Puthucheary ZA, McNelly AS, Rawal J, et al. Rectus femoris cross-sectional area and muscle layer thickness: comparative markers of muscle wasting and weakness. Am J Respir Crit Care Med 2017; 195:136-138.

42. Lukaski HC, Kyle UG, Kondrup J. Assessment of adult malnutrition and prognosis with bioelectrical impedance analysis: phase angle and impedance ratio. Curr Opin Clin Nutr Metab Care 2017; 20:330-339.

43. Shibahashi K, Sugiyama K, Kashiura M, Hamabe $Y$. Decreasing skeletal muscle as a risk factor for mortality in elderly patients with sepsis: a retrospective cohort study. J Intensive Care 2017; 5:8. 